\title{
A!
}

This is an electronic reprint of the original article.

This reprint may differ from the original in pagination and typographic detail.

Del Ferraro, Gino; Aurell, Erik

\section{Dynamic message-passing approach for kinetic spin models with reversible dynamics}

Published in:

Physical Review E

DOI:

10.1103/PhysRevE.92.010102

Published: 01/01/2015

Document Version

Publisher's PDF, also known as Version of record

Please cite the original version:

Del Ferraro, G., \& Aurell, E. (2015). Dynamic message-passing approach for kinetic spin models with reversible dynamics. Physical Review E, 92(1), 1-7. [010102]. https://doi.org/10.1103/PhysRevE.92.010102

This material is protected by copyright and other intellectual property rights, and duplication or sale of all or part of any of the repository collections is not permitted, except that material may be duplicated by you for your research use or educational purposes in electronic or print form. You must obtain permission for any other use. Electronic or print copies may not be offered, whether for sale or otherwise to anyone who is not an authorised user. 


\title{
Dynamic message-passing approach for kinetic spin models with reversible dynamics
}

\author{
Gino Del Ferraro ${ }^{1}$ and Erik Aurell ${ }^{1,2,3}$ \\ ${ }^{1}$ Department of Computational Biology, AlbaNova University Centre, SE-106 91 Stockholm, Sweden \\ ${ }^{2}$ ACCESS Linnaeus Centre, KTH-Royal Institute of Technology, SE-100 44 Stockholm, Sweden \\ ${ }^{3}$ Departments of Information and Computer Science and Applied Physics and Aalto Science Institute, Aalto University, \\ P. O. Box 15400, FI-00076 Aalto, Finland
}

(Received 17 November 2014; published 21 July 2015)

\begin{abstract}
A method to approximately close the dynamic cavity equations for synchronous reversible dynamics on a locally treelike topology is presented. The method builds on (a) a graph expansion to eliminate loops from the normalizations of each step in the dynamics and (b) an assumption that a set of auxilary probability distributions on histories of pairs of spins mainly have dependencies that are local in time. The closure is then effectuated by projecting these probability distributions on $n$-step Markov processes. The method is shown in detail on the level of ordinary Markov processes $(n=1)$ and outlined for higher-order approximations $(n>1)$. Numerical validations of the technique are provided for the reconstruction of the transient and equilibrium dynamics of the kinetic Ising model on a random graph with arbitrary connectivity symmetry.
\end{abstract}

DOI: 10.1103/PhysRevE.92.010102

PACS number(s): 02.50.-r, 89.20.-a

\section{INTRODUCTION}

Disordered spin systems are an important class of models able to catch and reproduce a large range of phenomena from phase transitions in magnets and amorphous systems [1] to protein folding in biology [2], social media [3,4], epidemic spreading [5], immune and neural networks [6], and applications in finance and optimization problems [7-9]. In the thermodynamic limit these systems have rich and fascinating repertoires of static and dynamic behavior, including the clustering [8] or shattering [10] transition, ergodicity breaking, and ageing [11]. To systematically describe their static properties the replica method (for fully connected systems) and the cavity method (for dilute systems) were developed [12]. General techniques to systematically study the dynamics of single finite systems in this class have, however, been less developed and in practice mostly limited to dynamic mean-field theories [13,14], path integral techniques [15-18], large deviation approaches [19], and, above all, numerical simulation [20].

The cavity method $[12,21]$ here holds a special place as it has become the method of choice to solve the statics of models on sparse networks, while for dynamics it was long restricted to dynamics on fully asymmetric graphs [22-24]. The main problem is that while the cavity technique reduces the complexity "in space" (number of terms in an approximate computation of marginals), there remains a complexity "in time" (cardinality of each term). This is so because the natural variables of the dynamic cavity method are probabilities of spin histories, of which there are exponentially many $\left(2^{t}\right.$ for synchronous updates over time $[0, t]$ with time constant one). Therefore, although the dynamic cavity equations themselves only involve a finite number of terms, summing them nevertheless (in general) entails a number of operations which is exponentially large in $t$. Fully asymmetric networks is a special case since the cavity equations then can be marginalized over time with no loss of information, and the complexity "in time" disappears. Alternative "ways out" investigated in the literature use additional assumptions on the evolution law such as majority dynamics [25] (i.e., linear dynamics with thresholding) or, more recently, unidirectional dynamics. Models of this latter type, where after a variable makes a transition from one state to another it can never go back, are represented by the zero-temperature random-field Ising model (RFIM) [26], cascade processes [27], spread optimization problems [28], and several epidemic models as, for instance, the susceptible-infected-recovered (SIR) model $[29,30]$. The main peculiarity of such models is that the dynamics can be parametrized in terms of the time(s) at which the transition from one state of the variable to another occurs, which again eliminates time complexity.

Another approach was taken in Ref. [31], where a simplifying "one-time" assumption was introduced, which for fully asymmetric networks reduces to (exact) marginalization over time. For the kinetic Ising model with synchronous and asynchronous updates this was later shown to be considerably more accurate than dynamic mean field, not only for asymmetric networks but also for partly symmetric networks [32,33]. A different approach, based on variational approximations, was very recently proposed in Ref. [34] where the author shows better performances in recovering stationary states compared to existing methods. Unfortunately, except for fully asymmetric networks, all these approaches are limited to steady states and hence cannot handle dynamic phenomena.

In this contribution we present a method to approximatively close the dynamic cavity equations for synchronous updates with no assumptions on the underlying network and evolution law beyond that the network is locally treelike and the evolution law is Markov. Unlike other methods already present in the literature, our approach is built not only to recover stationary states but also potentially the transient, i.e., the out-of-equilibrium dynamics. The method is built on two ingredients. The first, which already appeared in this context in Ref. [28], is the use of the graph expansion technique of Ref. [12] to rewrite the probabilistic model in a way such that the underlying graph is explicitly locally treelike, and the standard cavity equations can be used. The second ingredient 
is the assumption that a set of auxilary probability distributions on spin histories, "messages" in cavity method language, contain dependencies that are mainly local in time. A closure of the dynamic cavity equations is then effectuated within the class of $n$-order Markov processes. For definiteness we will here present the closure in the class of ordinary Markov processes $(n=1)$ and only outline the extensions to $n>1$, to which we intend to return in a future contribution. The pioneering contribution [31] can, in the present perspective, be seen as a closure in the class of Bernoulli processes $(n=0)$, without using graph expansion.

\section{THE DYNAMIC CAVITY EQUATIONS}

We consider a probabilistic graphical model defined on a treelike graph $\mathcal{G}=(V, E)$, where $V$ is a set of $N$ vertices and $E$ is a set of directed edges. Spins, Boolean variables $\sigma_{i}(t)=$ \pm 1 , are associated to each vertex at each time. We denote by $X_{i}=\left[\sigma_{i}(0), \sigma_{i}(1), \ldots, \sigma_{i}(t)\right]$ the spin history of spin $i$ and the evolution law $w_{i}\left(\sigma_{i}^{s} \mid \sigma_{i}^{s-1},\left\{\sigma_{j}^{s-1}\right\}_{j \in \partial i}\right)$ is the conditional probability of spin $i$ to take value $\sigma_{i}$ at time $s$ given the values of spins $i$ and $\partial i$, the graph neighbors of $i$, at time $s-1$. We note that this class is larger than the previously investigated majority dynamics and (synchronous) kinetic Ising models since we allow the evolution law to depend on $\sigma_{i}^{s-1}$. The joint probability over the spin histories can be then written as

$$
P\left(X_{1}, \ldots, X_{N}\right)=\prod_{i \in V} \prod_{s=1}^{t} w_{i}\left(\sigma_{i}^{s} \mid \sigma_{i}^{s-1},\left\{\sigma_{j}^{s-1}\right\}_{j \in \partial i}\right) P_{0}
$$

where $P_{0}=P\left[\sigma_{1}(0), \ldots, \sigma_{N}(0)\right]$ is the initial joint distribution at time zero and $t$ is the final time. We recall that the cavity method works well when the underlying network is (locally) treelike. Being a conditional probability, any evolution law $w_{i}$ can be written as $\exp \left(\sigma_{i}^{s} \Psi_{i}-\log 2 \cosh \Psi_{i}\right)$, where $\Psi_{i}$ is some function of $\left(\sigma_{i}^{s-1},\left\{\sigma_{j}^{s-1}\right\}\right)$. $w_{i}$ hence contains two types of "interactions," namely $\sigma_{i}^{s} \Psi_{i}$ and $\log 2 \cosh \Psi_{i}$, and if $P\left(X_{1}, \ldots, X_{N}\right)$ in (1) is written as $\exp \left[F\left(X_{1}, \ldots, X_{N}\right)\right]$, then the graph describing the dependencies in $F$ will have short loops, which necessarily emerge even if the network topology is treelike, as illustrated in Fig. 1. As shown in Refs. [12,28] such loops can however be removed by defining an auxiliary factor graph at the price that the new variable nodes will contain more that one old variable. The procedure consists in changing the old variable nodes into factor nodes and changing the old interaction edges into new variable nodes. The resulting topology of the new expanded graph is shown in Fig. 2, where each variable node now contains the spin histories of two spins. The functions sitting on the new factor nodes are defined in order to guarantee all interactions to remain the same as in the original graph and so, for consistency, the spin variables of the same type that appear on different (new) variable nodes have to take the same value. In the auxiliary graph the node $(i, j)$ contains the pair $\left[X_{i}^{(i j)}, X_{j}^{(i j)}\right]$, where by $X_{i}^{(i j)}$ we mean a variable of the same kind as spin history $X_{i}$ residing in the node $(i, j)$. The joint probability distribution of the new variables on

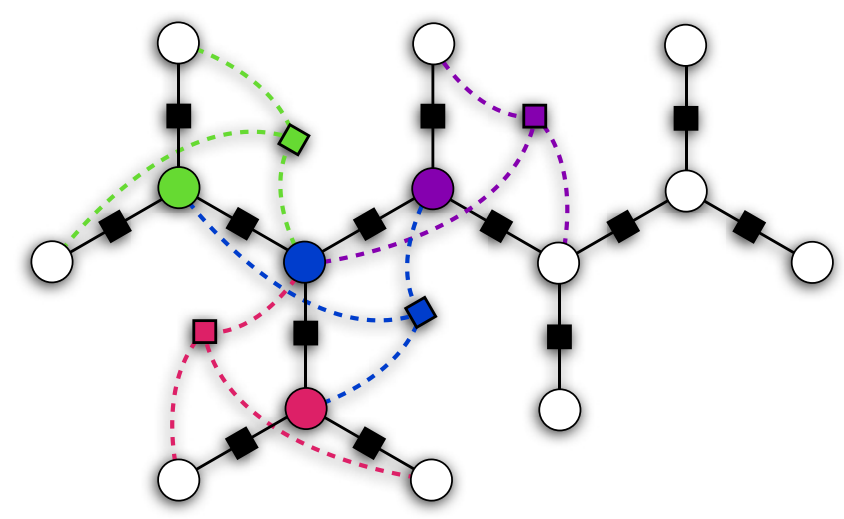

FIG. 1. (Color online) Original graph topologically treelike shown in a factor graph representation in which loops emerge naturally in time when dynamics is considered. Circles illustrate spin variables and squares interactions among them. More specifically, black squares indicate the interaction between one spin and one of its neighbors, i.e., $\phi_{i j}\left[\sigma_{i}(t), \sigma_{j}(t-1)\right]$, whereas colored squares show the interaction among neighbors of a given spin, i.e., $\phi_{j}\left[\left\{\sigma_{j}(t-1)\right\}_{j \in \partial i}\right]$.

the expanded factor graph of Fig. 2 then can be written as:

$$
\begin{aligned}
& P\left(\left\{X_{i}^{(i j)}, X_{j}^{(i j)}\right\}_{(i j) \in E}\right) \\
& \quad=\prod_{i \in V} \delta_{X_{i}^{\left(i j_{1}\right)}, X_{i}^{\left(i j_{2}\right)}, \ldots, X_{i}} \prod_{s=1}^{t} w_{i}\left(\sigma_{i}^{s} \mid \sigma_{i}^{s-1},\left\{\sigma_{j}^{s-1}\right\}_{j \in \partial i}\right) P_{0}
\end{aligned}
$$

where all the variables in $w_{i}\left(\sigma_{i}^{s} \mid \sigma_{i}^{s-1},\left\{\sigma_{j}^{s-1}\right\}_{j \in \partial i}\right)$ are taken from the surrounding spin histories $\left[X_{i}^{\left(i j_{1}\right)}, X_{i}^{\left(i j_{2}\right)}, \ldots\right]$ and the constraint enforces that these histories agree. If the original graph is (locally) treelike, then this procedure removes the loops in time and gives us a new auxiliary graph which is also (locally) treelike. The standard belief propagation (BP) update equations then can be written, for the variables in the new

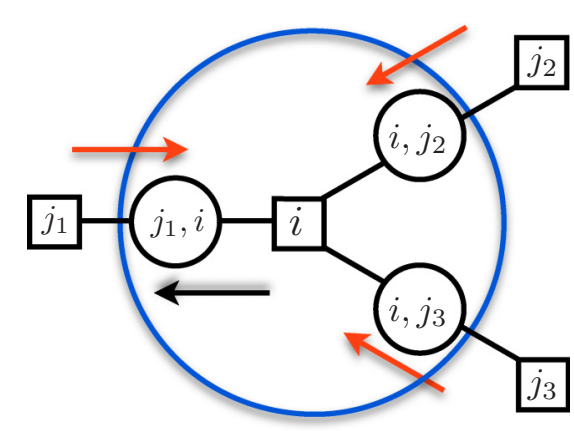

FIG. 2. (Color online) Auxiliary graph obtained from the original one shown in Fig. 1 where the time loops have been removed with a standard procedure. The new variable nodes contain the spin history of two variables which were neighbors in the original graph and factor nodes contain the interactions needed to generate and pass this history, i.e., $\tilde{\Phi}=$ $\delta_{X_{i}^{\left(i j_{1}\right)}, \ldots, X_{i}} \prod_{s=1}^{t} w_{i}\left(\sigma_{i}^{s} \mid \sigma_{i}^{s-1},\left\{\sigma_{j}^{s-1}\right\}_{j \in \partial i}\right) P_{0}\left(\sigma_{i},\left\{\sigma_{j}\right\}_{j \in \partial i}\right)$. Observe that the $\delta$ function is already summed over in the main text. 
graph, as in the static case (see Eq. (14.15) in Ref. [12]) as:

$$
m_{i \rightarrow(i j)}\left(X_{i}, X_{j}\right) \propto \sum_{\left\{X_{k}\right\}} \Phi\left(X_{i}, X_{j},\left\{X_{k}\right\}\right) \prod_{k \in \partial i \backslash j} m_{k \rightarrow(i k)}\left(X_{k}, X_{i}\right),
$$

where $\Phi\left(X_{i}, X_{j},\left\{X_{k}\right\}\right)=P_{0} \prod_{s=1}^{t} w_{i}\left(\sigma_{i}^{s} \mid \sigma_{i}^{s-1},\left\{\sigma_{k}^{s-1}\right\}_{k \in \partial i \backslash j}\right.$, $\left.\sigma_{j}^{s-1}\right)$ is analogous to a potential sitting in the factor nodes which transfers the dynamics to its neighbor variable nodes and the normalization factor can be computed by the condition that $\sum_{X_{i}, X_{j}} m_{i \rightarrow(i j)}\left(X_{i}, X_{j}\right)=1$. Above we have shortened the notation to $X_{i}=X_{i}^{(i j)}, X_{k}=X_{k}^{(i k)}$ and the same for $X_{j}$ and all the $\sigma$ variables contained in $w_{i}$. Let us note that we have fewer distinct messages with respect to the static BP formulation since messages from factor to variable nodes are, in this case, the same as messages from variable to factor nodes, i.e., $m_{j \rightarrow(i j)}\left(X_{j}, X_{i}\right)=m_{(i j) \rightarrow i}\left(X_{j}, X_{i}\right)$. Hence, for the topology shown in Fig. 2, the message on the left-hand side of (3) is illustrated in black, whereas the messages on the right-hand side are those two in red coming from the right side of the picture. To simplify notation, from now on variables with no apex refer to variables at time $t$, i.e., $\sigma_{i}=\sigma_{i}(t)$, whereas variables with apex(es) refer respectively to previous time(s), i.e., $\sigma_{i}^{\prime}=\sigma_{i}(t-1), \sigma_{i}^{\prime \prime}=\sigma_{i}(t-2), \ldots$, and so $X_{i}^{\prime}$ is the spin history of spin $i$ up to time $t-1$. Let us observe that, while notationally compact, Eq. (3) is, except for short times, computationally intractable as the right-hand side involves sums over complete spin histories.

\section{MARKOVIAN CLOSURE OF THE DYNAMIC CAVITY EQUATIONS}

We first observe that, given the Markovianity of the dynamics contained in $\Phi\left(X_{i}, X_{j},\left\{X_{k}\right\}\right)$, the messages $m_{i \rightarrow(i j)}\left(X_{i}, X_{j}\right)$ in (3) actually do not depend on the spin variable $\sigma_{j}$ at time $t$ but only at time $t-1$ and earlier times. The messages $m_{i \rightarrow(i j)}\left(X_{i}, X_{j}\right)$ are hence always uniform distributions on $\sigma_{j}(t)$ and, since $\sigma_{j}(t)$ is therefore a kind of dummy argument, we may simplify the notation by writing $m_{i \rightarrow(i j)}\left(X_{i}, X_{j}^{\prime}\right)$. Furthermore, if we use the same simplification on the right-hand side of (3), then we have incoming messages $m_{k \rightarrow(i k)}\left(X_{k}, X_{i}^{\prime}\right)$ which can be marginalized to $m_{k \rightarrow(i k)}\left(X_{k}^{\prime}, X_{i}^{\prime}\right)$ as there is no other dependence on $\sigma_{k}$, the value of spin $k$ at time $t$. Using Markovianity again we can simplify further to $m_{k \rightarrow(i k)}\left(X_{k}^{\prime}, X_{i}^{\prime \prime}\right)$ and write (3) as

$$
m_{i \rightarrow(i j)}\left(X_{i}, X_{j}^{\prime}\right) \propto \sum_{\left\{X_{k}^{\prime}\right\}} \Phi\left(X_{i}, X_{j}^{\prime},\left\{X_{k}^{\prime}\right\}\right) \prod_{k \in \partial i \backslash j} m_{k \rightarrow(i k)}\left(X_{k}^{\prime}, X_{i}^{\prime \prime}\right) .
$$

Equation (4) shows that the dynamic cavity equations have a different structure and are actually, in some respects, simpler than standard BP updates, since if all messages up to some time $t-1$ are known, then messages up to time $t$ can be evaluated directly without the need to iterate to a fixed point.

\section{A. Fully asymmetric graph}

It is worth observing how Eq. (4) simplifies when referred to a fully asymmetric graph, with interaction couplings between node $i$ and $j$ such that $J_{i j} \neq 0$ and $J_{j i}=0$. Under this assumption the interaction function $\Phi$ no longer depends on the spin history $X_{j}^{\prime}$ and, as a consequence, the message on the left-hand side of the equation does not depend on that history either, since such dependence is carried in only through the function $\Phi$. For consistency, we can apply the same argument to the messages on the right-hand side and conclude that they only depend on the spin history $X_{k}^{\prime}$ and no longer on $X_{i}^{\prime \prime}$. Then, remembering that $\Phi$ is a normalized function with respect to the variable $\sigma_{i}^{s}$, as shown in the text below Eq. (3), we can sum both sides of (4) over the spin history $X_{i}^{\prime}$ and then make use of the sum over $\left\{X_{k}^{\prime}\right\}$ on the right-hand side to obtain the simplified version of the dynamic message-passing equation for a fully asymmetric graph,

$$
\begin{aligned}
m_{i \rightarrow(i j)}\left(\sigma_{i}^{t}\right)= & \sum_{\left\{\sigma_{k}^{t-1}\right\}_{k \in \partial i \backslash j}} w_{i}\left(\sigma_{i}^{t} \mid\left\{\sigma_{k}^{t-1}\right\}_{k \in \partial i \backslash j}\right) \\
& \times \prod_{k \in \partial i \backslash j} m_{k \rightarrow(i k)}\left(\sigma_{k}^{t-1}\right) .
\end{aligned}
$$

This equation is in agreement with the literature for the same graph topology [24,31,32].

\section{B. Graph with arbitrary connectivity symmetry}

In what follows, we propose a Markovian closure of the dynamic BP update equation (4) for a network with arbitrary connectivity symmetry. In the next section the same closure is used to derivate the dynamic $B P$ output equations. In a closure in the class of $n$ th order Markov processes we assume $m_{i \rightarrow(i j)}\left(X_{i}, X_{j}^{\prime}\right)=$ $\prod_{s=n}^{t} T_{i \rightarrow(i j)}^{(n)}\left(\sigma_{i}^{s} \mid \sigma_{i}^{s-1}, \sigma_{j}^{s-1}, \ldots, \sigma_{i}^{s-n}, \sigma_{j}^{s-n}\right)$ and solve (4) iteratively. We here consider $n=1$. The marginalizations over the last and last two times of the variable node $(i, j)$ are

$$
\begin{aligned}
& P_{i \rightarrow(i j)}^{(t-1)}\left(\sigma_{i}^{\prime}, \sigma_{j}^{\prime}\right)=\sum_{X_{i}^{\prime \prime}, X_{j}^{\prime \prime}} m_{i \rightarrow(i j)}\left(X_{i}^{\prime}, X_{j}^{\prime \prime}\right), \\
& P_{i \rightarrow(i j)}^{(t, t-1)}\left(\sigma_{i}, \sigma_{i}^{\prime}, \sigma_{j}^{\prime}\right)=\sum_{X_{i}^{\prime \prime}, X_{j}^{\prime \prime}} m_{i \rightarrow(i j)}\left(X_{i}, X_{j}^{\prime}\right),
\end{aligned}
$$

by assumption linked by

$$
P^{(t, t-1)}\left(\sigma_{i}, \sigma_{i}^{\prime}, \sigma_{j}^{\prime}\right)=T\left(\sigma_{i} \mid \sigma_{i}^{\prime}, \sigma_{j}^{\prime}\right) P^{(t-1)}\left(\sigma_{i}^{\prime}, \sigma_{j}^{\prime}\right),
$$

where we omitted the subscript $i \rightarrow(i j)$ and the superscript time dependence of $T^{(t, t-1)}$ for readability. We note that, by the above, $P^{(t-1)}\left(\sigma_{i}^{\prime}, \sigma_{j}^{\prime}\right)$ actually does not depend on its second argument and we will therefore from now on simplify to $P^{(t-1)}\left(\sigma_{i}^{\prime}\right)$. Closure means to make the same assumptions for the upstream messages $m_{k \rightarrow(i k)}\left(X_{k}, X_{i}^{\prime}\right)$, use (4) to compute $P_{i \rightarrow(i j)}^{(t-1)}$ and $P_{i \rightarrow(i j)}^{(t, t-1)}$ in (6) and (7), and then take (8) to define $T\left(\sigma_{i} \mid \sigma_{i}^{\prime}, \sigma_{j}^{\prime}\right)$. This can be done by introducing an auxiliary function $\mathcal{F}$,

$$
\begin{aligned}
\mathcal{F}_{i \rightarrow(i j)}^{(t-1)}\left(\sigma_{i}^{\prime},\left\{\sigma_{k}^{\prime}\right\}_{k \in \partial i \backslash j}\right) & \sum_{\left\{X_{k}^{\prime \prime}, X_{i}^{\prime \prime}, X_{j}^{\prime \prime}\right\}} \prod_{k \in \partial i \backslash j} m_{k \rightarrow(i k)}\left(X_{k}^{\prime}, X_{i}^{\prime \prime}\right) \\
& \times \prod_{s=1}^{t-1} w_{i}\left(\sigma_{i}^{s} \mid \sigma_{i}^{s-1},\left\{\sigma_{k}^{s-1}\right\}_{k \in \partial i \backslash j}, \sigma_{j}^{s-1}\right) P_{0},
\end{aligned}
$$


which is strictly a specific marginalization of the right-hand side of (4). From now on, we use the notation $\left\{\sigma_{k}\right\}_{\backslash}=$ $\left\{\sigma_{k}\right\}_{k \in \partial i \backslash j}$ and $\prod_{k \backslash}=\prod_{k \in \partial i \backslash j}$. In terms of (9) we have

$$
\begin{gathered}
P_{i \rightarrow(i j)}^{(t-1)}\left(\sigma_{i}^{\prime}\right) \propto \sum_{\left\{\sigma_{k}^{\prime}\right\}} \mathcal{F}_{i \rightarrow(i j)}^{(t-1)}\left(\sigma_{i}^{\prime},\left\{\sigma_{k}^{\prime}\right\}\right), \\
P_{i \rightarrow(i j)}^{(t, t-1)}\left(\sigma_{i}, \sigma_{i}^{\prime}, \sigma_{j}^{\prime}\right) \propto \sum_{\left\{\sigma_{k}^{\prime}\right\}} w_{i}\left(\sigma_{i} \mid \sigma_{i}^{\prime},\left\{\sigma_{k}^{\prime}\right\}_{\backslash}, \sigma_{j}^{\prime}\right) \mathcal{F}_{i \rightarrow(i j)}^{(t-1)}\left(\sigma_{i}^{\prime},\left\{\sigma_{k}^{\prime}\right\}\right) .
\end{gathered}
$$

On the other hand, by the (assumed) Markovianity of the upstream messages we can write an iterative equation in time for $\mathcal{F}$ :

$$
\begin{aligned}
& \mathcal{F}_{i \rightarrow(i j)}^{(t)}\left(\sigma_{i},\left\{\sigma_{k}\right\}_{\backslash}\right) \\
& =\sum_{\left\{\sigma_{k}^{\prime}\right\}, \sigma_{i}^{\prime}, \sigma_{j}^{\prime}} \prod_{k \backslash} T_{k \rightarrow(i k)}\left(\sigma_{k} \mid \sigma_{k}^{\prime}, \sigma_{i}^{\prime}\right) w_{i}\left(\sigma_{i} \mid \sigma_{i}^{\prime},\left\{\sigma_{k}^{\prime}\right\}_{\backslash}, \sigma_{j}^{\prime}\right) \\
& \quad \times \mathcal{F}_{i \rightarrow(i j)}^{(t-1)}\left(\sigma_{i}^{\prime},\left\{\sigma_{k}^{\prime}\right\}_{\backslash}\right) .
\end{aligned}
$$

Whereas the iterative equation for $T$, using (10) and (11), reads as

$$
\begin{aligned}
& T_{i \rightarrow(i j)}\left(\sigma_{i} \mid \sigma_{i}^{\prime}, \sigma_{j}^{\prime}\right) \\
& =\frac{\sum_{\left\{\sigma_{k}^{\prime}\right\}} w_{i}\left(\sigma_{i} \mid \sigma_{i}^{\prime},\left\{\sigma_{k}^{\prime}\right\}_{\backslash}, \sigma_{j}^{\prime}\right) \mathcal{F}_{i \rightarrow(i j)}^{(t-1)}\left(\sigma_{i}^{\prime},\left\{\sigma_{k}^{\prime}\right\}_{\backslash}\right)}{\sum_{\sigma_{i},\left\{\sigma_{k}^{\prime}\right\}} w_{i}\left(\sigma_{i} \mid \sigma_{i}^{\prime},\left\{\sigma_{k}^{\prime}\right\}_{\backslash}, \sigma_{j}^{\prime}\right) \mathcal{F}_{i \rightarrow(i j)}^{(t-1)}\left(\sigma_{i}^{\prime},\left\{\sigma_{k}^{\prime}\right\}_{\backslash}\right)},
\end{aligned}
$$

where the denominator takes care of the normalization. Equations (12) and (13), which solve the dynamic cavity equations under the assumption that messages are Markovian, are the first result of this paper and represent the ingredients to solve the BP update equations at any time. The entire procedure clearly takes polynomial time instead of exponential time as the original formulation and, as noted above, does not involve iteration to a fixed point.

\section{MARKOVIAN CLOSURE OF THE BP OUTPUT EQUATIONS}

We now turn to the BP output equations, i.e., the equations for the actual marginal probability both on the auxiliary and on the original factor graph. The marginal probability of one variable node $(i, j)$ in the auxiliary graph is simply given by the product of the incoming messages to the node $(i, j)$ :

$$
P_{(i j)}\left(X_{i}, X_{j}\right) \propto m_{i \rightarrow(i j)}\left(X_{i}, X_{j}^{\prime}\right) m_{j \rightarrow(i j)}\left(X_{j}, X_{i}^{\prime}\right) .
$$

We are, however, interested in the single-site one-time marginal probability on the original graph, $P_{i}\left[\sigma_{i}(t)\right]$, from which we can compute physical observables of interest such as, for instance, the magnetization at any given time. We can get this marginal starting from the one-site marginal probability on a spin history, $P_{i}\left(X_{i}\right)$. In terms of the auxiliary probability distribution on the expanded graph this reads as

$$
\begin{aligned}
P_{i}\left(X_{i}\right) & \propto \sum_{X_{j}} \prod_{j \in \partial i} m_{j \rightarrow(i j)}\left(X_{j}, X_{i}^{\prime}\right) \\
& \times\left[\prod_{s=1}^{t} w_{i}\left(\sigma_{i}^{s} \mid \sigma_{i}^{s-1},\left\{\sigma_{j}^{s-1}\right\}_{j \in \partial i}\right) P_{0}\right],
\end{aligned}
$$

where spins $j$ 's are the neighbors of site $i$ in the original and expanded graph (see Fig. 2). To solve these equations on the same level of approximation as the update equation we define a new auxiliary function:

$$
\begin{aligned}
\mathcal{G}^{(t-1)}\left(\sigma_{i}^{\prime},\left\{\sigma_{j}^{\prime}\right\}_{j \in \partial i}\right)= & \sum_{X_{i,}^{\prime \prime}\left\{X_{j}^{\prime \prime}\right\}_{j \in \partial i}} \prod_{j \in \partial i} m_{j \rightarrow(i j)}\left(X_{j}^{\prime}, X_{i}^{\prime \prime}\right) \\
& \times\left[\prod_{s=1}^{t-1} w_{i}\left(\sigma_{i}^{s} \mid \sigma_{i}^{s-1},\left\{\sigma_{j}^{s-1}\right\}_{j \in \partial i}\right) P_{0}\right] .
\end{aligned}
$$

The single-site one-time and two-time marginal probabilities on the original graph then follow from (15):

$$
\begin{gathered}
P_{i}^{(t-1)}\left(\sigma_{i}^{\prime}\right) \propto \sum_{\left\{\sigma_{j}^{\prime}\right\}_{j \in \partial i}} \mathcal{G}^{(t-1)}\left(\sigma_{i}^{\prime},\left\{\sigma_{j}^{\prime}\right\}_{j \in \partial i}\right), \\
P_{i}^{(t, t-1)}\left(\sigma_{i}, \sigma_{i}^{\prime}\right) \propto \sum_{\left\{\sigma_{j}^{\prime}\right\}_{j \in \partial i}} w_{i}\left(\sigma_{i} \mid \sigma_{i}^{\prime},\left\{\sigma_{j}^{\prime}\right\}_{j \in \partial i}\right) \mathcal{G}^{(t-1)}\left(\sigma_{i}^{\prime},\left\{\sigma_{j}^{\prime}\right\}_{j \in \partial i}\right) .
\end{gathered}
$$

In analogy to $\mathcal{F}$ above, we can write a recursive equation for $\mathcal{G}$ by using the Markovian assumption for messages:

$$
\begin{aligned}
\mathcal{G}^{(t)}\left(\sigma_{i},\left\{\sigma_{j}\right\}_{j \in \partial i}\right)= & \sum_{\sigma_{i}^{\prime},\left\{\sigma_{j}^{\prime}\right\}_{j \in \partial i}} \prod_{j \in \partial i} T_{j \rightarrow(i j)}\left(\sigma_{j} \mid \sigma_{j}^{\prime}, \sigma_{i}^{\prime}\right) \\
& \times w_{i}\left(\sigma_{i} \mid \sigma_{i}^{\prime},\left\{\sigma_{j}^{\prime}\right\}_{j \in \partial i}\right) \mathcal{G}^{(t-1)}\left(\sigma_{i}^{\prime},\left\{\sigma_{j}^{\prime}\right\}_{j \in \partial i}\right) .
\end{aligned}
$$

Hence starting with an initial value for the functions $T, \mathcal{F}$, and $\mathcal{G}$, Eqs. (12), (13), and (19) can be iterated up to the desired time and Eq. (17) can be used to compute the timedependent marginal probability of site $i$. We highlight that, unlike the original formulation, which takes an exponential time to compute marginals, the entire scheme presented here has a polynomial computational cost.

\section{RESULTS}

In this section we test the accuracy of our dynamic messagepassing (DMP) approach on a statistical physics model often chosen as a case of study to investigate dynamics of complex systems: the kinetic Ising model.

\section{Case study: The kinetic Ising model with arbitrary connectivity symmetry}

We compare the performance of DMP to Monte Carlo Markov-chain simulations (MC) with Glauber dynamics on the kinetic Ising model on a random diluted graph with arbitrary connectivity symmetry: fully symmetric, partially asymmetric, and fully asymmetric networks. The comparison is performed computing the behavior of the magnetization of the model both during the transient and at equilibrium (or at the stationary states when detailed balance does not hold). Following Ref. [35], we introduce a connectivity matrix $c_{i j}$, where $c_{i j}=1$ if there is a link from vertex $i$ to vertex $j, c_{i j}=$ 0 otherwise, and matrix elements $c_{i j}$ and $c_{k l}$ are independent unless $\{k l\}=\{j i\}$. The following distributions then specify 
(a)

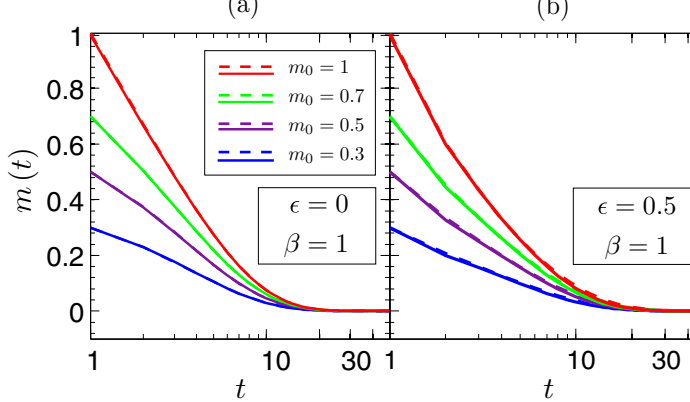

(c)

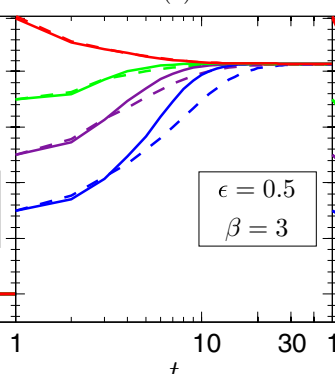

(d)

)

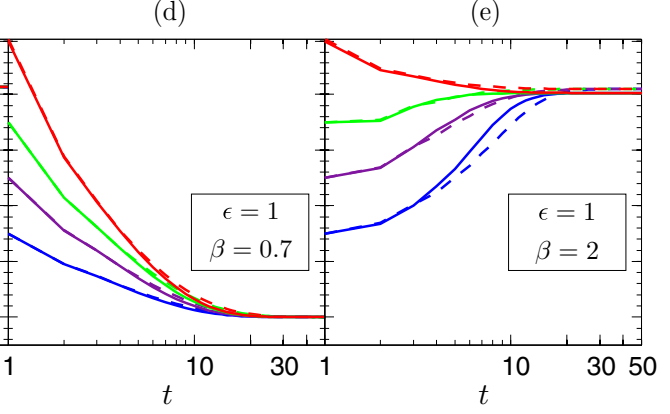

FIG. 3. (Color online) Comparison between dynamic message passing algorithm (solid lines) and Monte Carlo simulations (dashed lines) for the evolution of the magnetization $m(t)$ in an Erdös-Rényi network of $N=5000$ nodes and average connectivity $c=3$. Different colors represent different initial magnetizations $m(0)$, from the largest (upper line) to the smallest (lower line) according to the legend. (a) Fully asymmetric networks at high temperatures. [(b) and (c)] Partially asymmetric network $(\epsilon=0.5)$ respectively at high and low temperatures. [(d) and (e)] Fully symmetric network respectively at high and low temperatures. In MC simulations $m(t)$ is averaged over 5000 samples.

the graph topology: the marginal one-link distribution

$$
p\left(c_{i j}\right)=\frac{c}{N} \delta_{1, c_{i j}}+\left(1-\frac{c}{N}\right) \delta_{0, c_{i j}}
$$

and the conditional distribution

$$
p\left(c_{i j} \mid c_{j i}\right)=\epsilon \delta_{c_{i j}, c_{j i}}+(1-\epsilon) p\left(c_{i j}\right) .
$$

Above, $N$ is the size of the network, $c$ the average connectivity, and $\epsilon \in[0,1]$ a parameter which controls the asymmetry. The value $\epsilon=0$ gives a fully asymmetric network, whereas $\epsilon=1$ gives a fully symmetric one.

In the kinetic Ising model the transition probability rate $w_{i}$, which appears above, is given by $w_{i}\left(\sigma_{i}^{s} \mid\left\{\sigma_{j}^{s-1}\right\}\right)=$ $\exp \left[\beta \sigma_{i}^{s} J_{i j} \sigma_{j}^{s-1}-\log 2 \cosh \left(\beta \sum_{j} J_{i j} \sigma_{j}^{s-1}\right)\right]$, where the $j$ 's variable are neighbors of spin $i, J_{i j}$ are the interaction strengths between sites $i$ and $j$, and $\beta$ is the inverse temperature. We observe that this model belongs to a subclass of the models considered within the general formulation above as the transition rate does not depend explicitly on $\sigma_{i}^{s-1}$. Since we here want to mainly investigate the effect of the asymmetry on the performances of our algorithm, we restrict our numerical analysis to ferromagnetic models, i.e., interaction strength $J_{i j}=J>0$ for every pair of sites $i, j$. For simplicity, all the spins on the graph are chosen to be independent at the initial time although correlated initial conditions could also be considered in the above formulation. Results are shown in Fig. 3 for several values of the initial magnetization at different temperatures and for various values of the asymmetry parameter $\epsilon$. Numerical results for fully asymmetric networks are obtained by using the simplified version (5) of the dynamic message-passing equation and, as expected from Refs. [31-33], they show a perfect agreement with Monte Carlo simulations both for the computation of the transient and the stationary state [see Fig. 3(a)]. When the full asymmetry is broken and a network with feedback is considered, numerics is obtained through the DMP scheme presented above. Comparison with MC shows that the results provided by DMP are still very good for high-enough temperature (above the critical transition) both for partially and fully symmetric connectivities. Indeed, as it is possible to note from Fig. 3 [partially asymmetric network in Fig. 3(b) and the fully symmetric one in Fig. 3(d)], the transient regime is very well reproduced by DMP and the stationary (or equilibrium) state is the same as MC. When temperature is decreased below the critical ferromagnetic transition the performances of DMP begin to worsen [Figs. 3(c) and 3(e)]. In this regime, the transient is very well recovered only for the first few initial steps of the dynamics and progressively worsen for longer times. Nevertheless, for the partially asymmetric network considered $(\epsilon=0.5)$ the stationary state reached by the DMP and MC simulations coincide, although DMP has a faster convergence to that [Fig. 3(c)]. For fully symmetric networks similar considerations follow for the transient regime although the equilibrium state reached by the DMP and MC simulations at this temperature slightly differ [Fig. 3(e)]. This is not always the case; indeed, we noticed numerically that, for some of the temperatures even below the critical transition, DMP reaches the same equilibrium state as $\mathrm{MC}$ [see, for instance, Fig. 4(c)]. However, for fully symmetric networks, it is known that the stationary solutions of the one-time approximation (OTA) method presented in Refs. [24,31] are also the solution of the static BP equations. Therefore the agreement between the MC simulations and the one-time approximation for the equilibrium state of fully symmetric networks is expected to be near perfect, whereas, for some temperatures, the DMP approach proposed here presents small differences with the equilibrium MC solution.

In order to investigate the performances of DMP with respect to the one-time approximation, we compared both algorithms with MC simulations for different temperatures and for various values of the asymmetry parameter $\epsilon$. The numerical investigation, partially illustrated in Fig. 4, shows that, regardless, the network connectivity symmetry DMP always outperforms OTA for the transient dynamic regime. For temperatures above the critical transition $\left(T_{c}\right)$ both algorithms converge to the same stationary or equilibrium state, in agreement with the MC simulations [see Figs. 4(a) and 4(d)] and, surprisingly, the one-time approximation recovers well the MC stationary solution also for partially asymmetric networks [Fig. 4(d)]. For temperatures lower than $T_{c}$ the following picture emerges. For fully symmetric networks OTA always reaches the same equilibrium state as MC, whereas DMP in some cases does [see Fig. 4(c)] and in some cases 
(a)

(b)

(c)

(d)

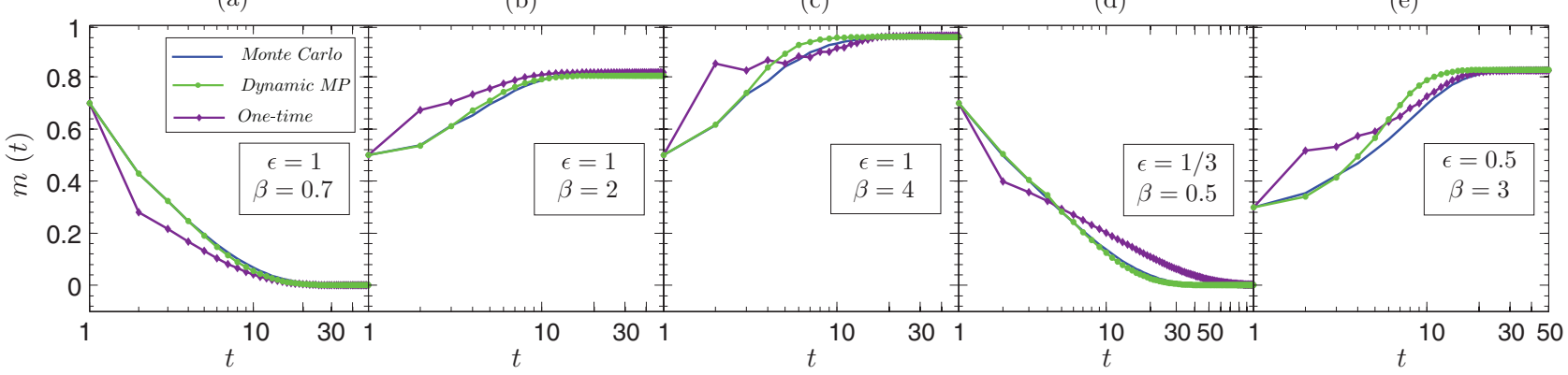

FIG. 4. (Color online) Comparison among the dynamic message passing algorithm (solid lines with • dots), the one-time approximation (solid lines with dots), and the Monte Carlo simulations (solid lines) for the evolution of the magnetization $m(t)$ in an in an Erdös-Rényi network of $N=5000$ nodes and average connectivity $c=3$. [(a)-(c)] Fully symmetric network at high and low temperatures. [(d) and (e)] Partially symmetric networks with different connectivity symmetry at high and low temperatures. In the MC simulations $m(t)$ is averaged over 5000 samples.

does not [see Fig. 4(b)], depending on the temperature. For partially asymmetric networks, the performances of DMP get better and, surprisingly, OTA also gives fairly good results for the stationary states [see Fig. 4(d)]. The differences in the reconstruction of the transient and stationary regime of the DMP and the one-time approach can be understood, remembering that the two algorithms do not belong to the same class of approximations.

We conclude this section by underlining that we expect improvement of the results provided by the dynamic messagepassing scheme presented, both for the transient and for the stationary or equilibrium states, when a higher-order closure of the DMP equations is made (see the next section).

\section{HIGHER-ORDER CLOSURES}

To close (3) in the class of $n$-th order Markov processes, we consider, instead of $P_{i \rightarrow(i j)}^{(t-1)}$ and $P_{i \rightarrow(i j)}^{(t, t-1)}$ in (10) and (11), the marginalizations $P_{i \rightarrow(i j)}^{(t-1, t-2, \ldots, t-n)}$ and $P_{i \rightarrow(i j)}^{(t, t-1, t-2, \ldots, t-n)}$ and auxiliary functions $F^{(n)}$ and $G^{(n)}$ which depend on $n$ earlier times. The time iteration of $F^{(n)}, G^{(n)}$ and the iterative solution of the $n$ th-order kernel $T_{i \rightarrow(i j)}^{(n)}\left[\sigma_{i} \mid \sigma_{i}^{\prime}, \sigma_{j}^{\prime}, \ldots, \sigma_{i}(t-n), \sigma_{j}(t-\right.$ $n)]$ then proceed analogously as above. The computational cost obviously increases quickly with $n$ and the value of higherorder closures will depend on the application and the model. This issue will be addressed in future contributions.

\section{ACKNOWLEDGMENTS}

The authors acknowledge valuable discussions with Federico Ricci-Tersenghi, Silvio Franz, Haiping Huang, Andrey Y. Lokhov, Yoshiyuki Kabashima, and Gabriele Perugini. This work was finalized during the program "Collective Dynamics in Information System" at Kavli Institute for Theoretical Physics China (KITPC) in Beijing, China. It has been funded under FP7/2007-2013/Grant No.j 290038 (GDF) and supported by the Swedish Science Council through Grant No. 621-2012-2982 and by the Academy of Finland through its Center of Excellence COIN (E.A.).
[1] A. Crisanti and L. Leuzzi, Amorphous-amorphous transition and the two-step replica symmetry breaking phase, Phys. Rev. B 76, 184417 (2007).

[2] R. A. Goldstein, Z. A. Luthey-Schulten, and P. G. Wolynes, Optimal protein-folding codes from spin-glass theory, Proc. Natl. Acad. Sci. USA 89, 4918 (1992).

[3] G. Facchetti, G. Iacono, and C. Altafini, Computing global structural balance in large-scale signed social networks, Proc. Natl. Acad. Sci. USA 108, 20953 (2011).

[4] S. Kirkpatrick, A. Kulakovsky, M. Cebrian, and A. S. Pentland, Social networks and spin glasses, Philos. Mag. 92, 362 (2012).

[5] R. Pastor-Satorras and A. Vespignani, Epidemic spreading in scale-free networks, Phys. Rev. Lett. 86, 3200 (2001).

[6] D. J. Amit, Modeling Brain Function: The World of Attractor Neural Networks (Cambridge University Press, Cambridge, 1992).
[7] A. C. C. Coolen, The Mathematical Theory of Minority Games: Statistical Mechanics of Interacting Agents (Oxford Finance Series) (Oxford University Press, Oxford, 2005).

[8] M. Mézard, G. Parisi, and R. Zecchina, Analytic and algorithmic solution of random satisfiability problems, Science 297, 812 (2002).

[9] S. Franz, M. Leone, F. Ricci-Tersenghi, and R. Zecchina, Exact solutions for diluted spin glasses and optimization problems, Phys. Rev. Lett. 87, 127209 (2001).

[10] D. Achlioptas, Algorithmic barriers from phase transitions in graphs, in Graph Theoretic Concepts in Computer Science (Springer, Berlin, 2010), p. 1.

[11] J.-P. Bouchaud, L. F. Cugliandolo, J. Kurchan, and M. Mézard, Out of equilibrium dynamics in spin-glasses and other glassy systems, Spin Glasses and Random Fields 12, 161 (1997).

[12] M. Mezard and A. Montanari, Information, Physics, and Computation (Oxford University Press, Oxford, 2009). 
[13] H. J. Kappen and F. B. Rodriguez, Efficient learning in Boltzmann machines using linear response theory, Neural. Comput. 10, 1137 (1998).

[14] H. J. Kappen and J. J. Spanjers, Mean field theory for asymmetric neural networks, Phys. Rev. E 61, 5658 (2000).

[15] C. De Dominicis, Dynamics as a substitute for replicas in systems with quenched random impurities, Phys. Rev. B 18, 4913 (1978).

[16] H. Sompolinsky and A. Zippelius, Relaxational dynamics of the Edwards-Anderson model and the mean-field theory of spinglasses, Phys. Rev. B 25, 6860 (1982).

[17] H.-J. Sommers, Path-integral approach to Ising spin-glass dynamics, Phys. Rev. Lett. 58, 1268 (1987).

[18] Y. Roudi and J. Hertz, Dynamical TAP equations for nonequilibrium Ising spin glasses, J. Stat. Mech. (2011) P03031.

[19] G. Del Ferraro and E. Aurell, Perturbative large deviation analysis of non-equilibrium dynamics, J. Phys. Soc. Jpn. 83, 084001 (2014).

[20] D. T. Gillespie, Exact stochastic simulation of coupled chemical reactions, J. Phys. Chem. 81, 2340 (1977).

[21] J. S. Yedidia, W. T. Freeman, and Y. Weiss, Understanding belief propagation and its generalizations, Exploring Artificial Intelligence in the New Millennium (Morgan Kaufmann Publishers Inc., San Francisco, CA, USA 2003), pp. 239-269.

[22] B. Derrida, E. Gardner, and A. Zippelius, An exactly solvable asymmetric neural network model, Europhys. Lett. 4, 167 (1987).

[23] M. Mézard and J. Sakellariou, Exact mean-field inference in asymmetric kinetic Ising systems, J. Stat. Mech. (2011) L07001.

[24] E. Aurell and H. Mahmoudi, Three lemmas on dynamic cavity method, Commun. Theor. Phys. 56, 157 (2011).
[25] Y. Kanoria and A. Montanari, Majority dynamics on trees and the dynamic cavity method, Ann. Appl. Probab. 21, 1694 (2011).

[26] H. Ohta and S.-i. Sasa, A universal form of slow dynamics in zero-temperature random-field Ising model, Europhys. Lett. 90, 27008 (2010).

[27] F. Altarelli, A. Braunstein, L. Dall'Asta, and R. Zecchina, Large deviations of cascade processes on graphs, Phys. Rev. E 87, 062115 (2013).

[28] F. Altarelli, A. Braunstein, L. Dall'Asta, and R. Zecchina, Optimizing spread dynamics on graphs by message passing, J. Stat. Mech. (2013) P09011.

[29] A. Y. Lokhov, M. Mézard, H. Ohta, and L. Zdeborová, Inferring the origin of an epidemic with dynamic message-passing algorithm, Phys. Rev. E 90, 012801 (2014).

[30] A. Y. Lokhov, M. Mézard, and L. Zdeborová, Dynamic messagepassing equations for models with unidirectional dynamics, Phys. Rev. E 91, 012811 (2015).

[31] I. Neri and D. Bollé, The cavity approach to parallel dynamics of Ising spins on a graph, J. Stat. Mech. (2009) P08009.

[32] E. Aurell and H. Mahmoudi, A message-passing scheme for non-equilibrium stationary states, J. Stat. Mech. (2011) P04014.

[33] E. Aurell and H. Mahmoudi, Dynamic mean-field and cavity methods for diluted Ising systems, Phys. Rev. E 85, 031119 (2012).

[34] A. Pelizzola, Variational approximations for stationary states of Ising-like models, Eur. Phys. J. B 86, 120 (2013).

[35] J. P. L. Hatchett, B. Wemmenhove, I. Pérez Castillo, T. Nikoletopoulos, N. S. Skantzos, and A. C. C. Coolen, Parallel dynamics of disordered Ising spin systems on finitely connected random graphs, J. Phys. A: Math. Gen. 37, 6201 (2004). 